\title{
BMJ Open Observational longitudinal multicentre investigation of acute pancreatitis (GOULASH PLUS): follow-up of the GOULASH study, protocol
}

Alexandra Mikó, ${ }^{1}$ Bálint Erőss, ${ }^{1}$ Patrícia Sarlós, ${ }^{2}$ Péter Hegyi Jr, ${ }^{1,3}$ Katalin Márta, ${ }^{1,4}$ Dániel Pécsi, ${ }^{2}$ Áron Vincze, ${ }^{2}$ Beáta Bódis, ${ }^{5}$ Orsolya Nemes, ${ }^{5}$ Nándor Faluhelyi, ${ }^{6}$ Orsolya Farkas, ${ }^{6}$ Róbert Papp, ${ }^{7}$ Dezső Kelemen, ${ }^{7}$ Andrea Szentesi, ${ }^{1,8}$ Eszter Hegyi, ${ }^{1}$ Mária Papp, ${ }^{9}$ László Czakó, ${ }^{8}$ Ferenc Izbéki, ${ }^{10}$ László Gajdán, ${ }^{10}$ János Novák, ${ }^{11}$ Miklós Sahin-Tóth, ${ }^{12}$ Markus M Lerch, ${ }^{13}$ John Neoptolemos, ${ }^{14}$ Ole H Petersen, ${ }^{15}$ Péter Hegyi ${ }^{\odot 1,7,16}$

To cite: Mikó A, Erőss B, Sarlós P, et al. Observational longitudinal multicentre investigation of acute pancreatitis (GOULASH PLUS): follow-up of the GOULASH study, protocol. BMJ Open 2019;9:e025500. doi:10.1136/ bmjopen-2018-025500

- Prepublication history and additional material for this paper are available online. To view please visit the journal (http:// dx.doi.org/10.1136/bmjopen2018-025500).

Received 21 July 2018 Revised 19 February 2019 Accepted 2 May 2019

Check for updates

(C) Author(s) (or their employer(s)) 2019. Re-use permitted under CC BY-NC. No commercial re-use. See rights and permissions. Published by BMJ.

For numbered affiliations see end of article.

Correspondence to Dr Péter Hegyi; p.hegyi@tm-centre.org

\section{ABSTRACT}

Background Acute pancreatitis (AP) is an inflammatory condition that can lead to late consequences. Recurrent AP (RAP) develops in $20 \%$ of patients and chronic pancreatitis (CP) occurs in 7\%-12.8\%. However, we do not have sufficient information to establish an evidencebased statement to define early $\mathrm{CP}$, or how to prevent its development.

Aim The aim of this study was to understand the influencing factors and to determine which parameters should be measured or used as a biomarker to detect the early phase of CP.

Methods/Design This is an observational prospective follow-up study of the GOULASH-trial (ISRTCN 63827758) in which (1) all severity of pancreatitis are included; (2) patients receive only therapeutic modalities which are accepted by the evidence based medicine (EBM) guideline; (3) whole blood, serum and plasma samples are stored in our biobank; and (4) large amount of variables are collected and kept in our electronic database including anamnestic data, physical examination, laboratory parameters, imaging, therapy and complications. Therefore, this fully characterised patient cohort are well suitable for this longitudinal follow-up study. Patients' selection: patients enrolled in the GOULASH study will be offered to join to the Iongitudinal study. The follow-up will be at 1, 2, 3, 4, 5 and 6 years after the episode of AP. Anamnestic data will be collected by questionnaires: (1) diet history questionnaire, (2) 36-Item Short-Form Health Survey, (3) physical activity questionnaire and (4) stress questionnaire. Genetic tests will be performed for the genes associated with CP. The exocrine and endocrine pancreatic, liver and kidney functions will be determined by laboratory tests, stool sample analyses and imaging. Cost-effectiveness will be analysed to examine the relationship between events of interest and healthrelated quality of life or to explore subgroup differences. Conclusion This study will provide information about the risk and influencing factors leading to $\mathrm{CP}$ and identify the most useful measurable parameters.

\section{Strengths and limitations of this study}

- Patients suffering from acute pancreatitis (AP) will be followed up at 1, 2, 3, 4, 5 and 6 years after the episode of AP. This way the study is able to give a more precise, detailed insight of the possible development of pancreatitis within a wide time frame.

- Anamnestic data will be collected by questionnaires about dietary habits, physical activity, stress and anamnestic data questionnaires, which means plentiful quality data collection with low costs; therefore, the study has a satisfactory cost-benefit ratio.

- Genetic tests will be performed for deeper level of understanding of the genetic background of chronic pancreatitis (CP).

- Since the leading aetiology of CP is alcohol, several subjects may not attend the follow-up appointment every year.

- Considering that CP will be assessed on a yearly basis, we will have no exact date when the CP developed.

Trial registration number ISRCTN63396106

\section{BACKGROUND}

Acute pancreatitis (AP) is a severe inflammatory condition with possible late complications. ${ }^{1}$ Twenty per cent of patients with AP tend to develop recurrent acute pancreatitis (RAP), ${ }^{2}$ and in $7 \%-12.8 \%$ of patients, chronic pancreatitis (CP) will occur. ${ }^{3-5} \mathrm{CP}$ is an incurable disease; the incidence of $\mathrm{CP}$ in Western countries is $10-50 / 100000$ and it is more common in men. ${ }^{26}$ It is well documented that RAP can lead to CP; therefore, it might be considered as the early form of CP. From the first attack of AP until the 
end-stage CP, there is a long period; however, there is no information about the characteristic of the road, or how the CP will be formed from the first attack. Risk factors leading to CP or influencing the development of $\mathrm{CP}$ are not well characterised yet.

CP represents a chronic inflammatory state of the pancreas which is caused by regular alcohol consumption, smoking, hypercalcaemia and genetic factors. ${ }^{7}$ Alcohol consumption is the most common risk factor, ${ }^{89}$ which increases the risk logarithmically. ${ }^{10} 11$ The Harmonizing diagnosis and treatment of chronic pancreatitis across Europe (HaPanEu) guideline highlights that smoking is an independent risk factor which may worsen the prognosis of CP. ${ }^{11} \mathrm{CP}$ is most commonly diagnosed at late stages based on morphological changes; fibrosis can be observed and exocrine insufficiency is present, which means a pancreatic burnout with more than $90 \%$ loss of the healthy pancreatic tissue. ${ }^{12}$ Whitcomb formulated the definition of the early phase of CP but the parameters that could indicate the diagnosis are not well characterised. ${ }^{13}$ Yamabe et al highlighted in a review that the screening of patients for suspicion of early CP and the treatment of early CP are very important from both the patients' and the healthcare economics' point of view. ${ }^{14}$

An important feature of $\mathrm{CP}$ is pancreatic exocrine insufficiency (PEI), which leads to the deterioration of the nutritional status and worsen the quality of life of patients without treatment and follow-up. ${ }^{15} \mathrm{PEI}$ is also a risk factor for increased mortality in patients with $\mathrm{CP}^{16}$ Because of the fat malabsorption, steatorrhea will occur and measuring faecal elastase-1 level provides indirect information on exocrine pancreatic insufficiency. ${ }^{16}$ The gold standard treatment of PEI is pancreatic enzyme replacement therapy, which helps to avoid malnutrition and improves the nutritional status of patients with $\mathrm{CP}^{1718}$; therefore, patients with high risk for $\mathrm{CP}$ need to be followed and therapy should be initiated as early as possible.

Pancreatic endocrine insufficiency develops more frequently after severe AP and its severity is directly associated with the extent of pancreatic necrosis, ${ }^{19}$ and it is defined as type $3 c$ diabetes or pancreatogenic diabetes. In pancreatogenic diabetes, both insulin production and glucagon secretion are impaired. ${ }^{20}$ The importance of the topic is confirmed by the recently developed prediabetes self-assessment screening score after acute pancreatitis (PERSEUS) score, which helps to identify individuals who are at high risk for developing new-onset diabetes after an episode of AP. ${ }^{21}$

Most patients with CP are suffering from recurrent episodes of pain, which usually cannot be eliminated by conservative treatment. Based on the step-up approach, endoscopic interventions are given prior to surgery, but a meta-analysis showed that early surgery is associated with increased likelihood of complete postoperative pain relief, reduced risk of pancreatic insufficiency and the need for further intervention. ${ }^{22}$
Cost-effectiveness analysis is the evaluation of the joint distribution of differences in cost and clinical effect and has a very important role in chronic diseases. It provides an early opportunity to produce reliable estimates of cost-effectiveness at low marginal cost. Access to individual patient data also permits a wide range of statistical and econometric techniques to examine the relationship between events of interest and health-related quality of life or to explore subgroup differences.

Several studies analysed the changes in laboratory parameters in CP. The concentration of leptin ${ }^{23}$ was significantly lower in the serum samples of patients with CP compared with controls, while interleukin (IL) $1 \beta$, IL-6, IL- 8 and tumour necrosis factor $\alpha$ (TNF- $\alpha$ ) showed elevated levels. ${ }^{24}$ A review summarised the measurable parameters of the beta cell function, which can also be used in the diagnosis. ${ }^{25}$

Genetics plays an important role in the development of CP. As CP often evolves through recurrent attacks of $\mathrm{AP}$, in our study we aim to investigate the effect of genetic risk factors on the disease onset and progression. Over the past two decades, it has been demonstrated that premature trypsinogen activation plays a central pathogenic role in the development of $\mathrm{CP}^{26}$ Moreover, an alternative pathomechanism unrelated to accelerated intrapancreatic trypsinogen activation has been identified, in which mutation-induced misfolding and consequent endoplasmic reticulum stress lead to acinar cell damage and pancreatitis. ${ }^{26}$

\section{AIMS AND OBJECTIVES}

GOULASH PLUS is an observational prospective follow-up study of the individuals enrolled in the GOULASH trial ${ }^{27}$ (http://www.pancreas.hu/en/ studies/goulash, ISRTCN 63827758) in which (1) all severity of pancreatitis are included; (2) patients receive only therapeutic modalities which are part of the evidence-based guideline; (3) whole blood, serum and plasma samples of the enrolled individuals are stored in our biobank; and (4) a large number of variables are collected and kept in our electronic database, including medical and family history, findings of physical examination, laboratory parameters, results of diagnostic imaging and detailed data on therapy and complications. Therefore, the fully characterised patient cohort of the GOULASH trial is ideal for this longitudinal follow-up study.

Our aim is to understand the course of AP leading to $\mathrm{CP}$ and to find the factors that can protect the pancreas from the development of the disease. We will follow the most important factors in our subjects' structure, aiming to find protective factors and the first predictable parameters of the disease. The study will provide results, which will help to understand the development and risk factors of late complications in AP and the development of the pancreatic damage. 


\section{METHODS}

\section{Study design}

GOULASH PLUS is a 6-year observational prospective follow-up study with no intervention. Patients suffering from AP, who took part in the GOULASH study, will be approached and enrolled if consent is given. Participants will be followed up in 1, 2, 3, 4, 5 and 6 years following their discharge after the admission with AP. The follow-up visits will be arranged every 12 months $( \pm 1$ month) for 6 years. After receiving detailed verbal and written information on the study, the consent form will be signed. If consent is given, the following tests and investigations will be arranged at each follow-up, and we collect the information under the same code as used in the GOULASH study completed with $\mathrm{P}$ and the number of the year of the follow-up to avoid the contravention of personal rights.

To detect the earliest morphological changes and late complications of AP, transabdominal ultrasonography (US) (1, 3, 5 years) and in the second, fourth and sixth year endoscopic ultrasonography (EUS) examination will be performed (figure 2). The transabdominal US is part of the standard work-up for patients with $\mathrm{AP}^{28}$; however, several studies explored the visualisation of morphological changes in CP by the transabdominal US and reported varying diagnostic accuracy with a sensitivity of $70 \%-80 \% .{ }^{29} 30$ One large study reported sensitivities for US $>85 \%$ for the features calcifications, pancreatic duct dilations and cysts. ${ }^{30}$ Generally, EUS will be suggested based on the HaPanEU guideline's 2-9 point. ${ }^{11}$ It is the most sensitive imaging technique in the early phase of CP, but if the participant disagrees, MRI will be performed for increasing the attendance on the controls. A recent meta-analysis ${ }^{31}$ showed that the sensitivity of EUS and magnetic resonance cholangiopacreatography (MRCP) are $81 \%$ and $78 \%$, respectively, while the specificity was $90 \%$ and $96 \%$, respectively. The radiologist may indicate a CT, EUS or MRI examination if the pancreas is not visualisable, uncertain features of CP on the US scan, suspicion of focal lesions or any novum alterations in the pancreas. Blood samples will be collected for laboratory analysis and stored in biobank for later testing. Oral glucose tolerance test (OGTT) will be performed when diabetes mellitus (DM) is unknown, and fasting plasma glucose (FPG) level is $\leq 7 \mathrm{mmol} / \mathrm{L}$. In this trial, we will be guided by the diagnostic criteria of DM and prediabetic states, which were defined by the WHO in 1999. ${ }^{8}$ Homeostasis model assessment of $\beta$-cell function (HOMA-Beta) will be calculated based on $20 \times$ Insulin/ Glucose-3.5 equation in percentage. ${ }^{25}$ Patients will be given a stool sample collection kit and they will be asked to provide a sample for the measurement of faecal elastase level, which is the most widely employed non-invasive test to document EPI. Faecal elastase is widely available and can be performed while the patient is taking commercial pancreatic enzyme replacement therapy (PERT) products. The interpretation of faecal elastase test will be made based on normal pancreatic function 200-500 $\mathrm{\mu g} / \mathrm{g}$, mild pancreatic dysfunction 100-199 $\mu \mathrm{g} / \mathrm{g}$ and severe pancreas exocrine insufficiency $<100 \mu \mathrm{g} / \mathrm{g}$. For the detailed assessment of the participants' diet, the validated Diet History Questionnaire (DHQ) will be completed (table 1, online supplementary file 1). Pain, environmental factors such as alcohol consumption and smoking, physical activity and quality of life will be detected by validated questionnaires. To collect detailed information on the quality of life and pain, 36-Item Short-Form Health Survey (SF-36) questionnaire will be used (table 1, online supplementary file $1)$. The questionnaire will be completed with the help of a clinical research administrator; therefore, improperly completed datasheets and incorrect data upload will be avoided and controlled.

The diagnosis of CP is based on clinical (weight loss, malnutrition, diarrhoea), morphological (imaging) and functional parameters (pancreatic function tests). ${ }^{81132}$ The diagnosis will be based, at EUS examination, on morphological changes according to Rosemont criteria $^{33}$ (online supplementary file 2), on the presence of pancreatic ductal calcifications examined by US and CT, and pancreatic duct abnormalities investigated by MRCP. $^{11}$

Whenever these alterations have been diagnosed, CP is already developed; in this study, the early phase of CP will be investigated in the 2-year period before the CP retrospectively.

\begin{tabular}{ll}
\hline \multicolumn{1}{l}{ Table 1 Schedule of fulfilled questionnaires during the follow-up period } \\
\hline Title & Source \\
\hline $\begin{array}{l}\text { Physical and } \\
\text { socioeconomic status }\end{array}$ & $\begin{array}{l}\text { American Community Survey (ACS); The MacArthur Scale of Subjective Social Status } \\
\text { (Supplementary document 1 page 11-12) }\end{array}$ \\
$\begin{array}{l}\text { Details from the medical } \\
\text { history }\end{array}$ & $\begin{array}{l}\text { Acute Pancreatitis Questionnaire (Registry for Pancreatic Patients by Hungarian Pancreatic Study } \\
\text { Group) (Supplementary document 1 page 1-10) }\end{array}$ \\
Dietary habits & $\begin{array}{l}\text { Diet History Questionnaire, V.2.0. National Institutes of Health, Epidemiology and Genomics } \\
\text { Research Program, National Cancer Institute. 2010. (Supplementary document 1 page 28-68) }\end{array}$ \\
Physical activity & $\begin{array}{l}\text { International Physical Activity Questionnaire (IPAQ) (long, usual week version) (Supplementary } \\
\text { document 1 page 13-17) }\end{array}$ \\
Stress & Perceived Stress Scale (10-item version) (Supplementary document 1 page 17-18) \\
Quality of life, pain & RAND 36-Item Health Survey (Version 1.0) (SF-36) (Supplementary document 1 page 22-27) $^{30}$
\end{tabular}


Genetic testing of participants will be performed in the most important genes for AP and CP, such as PRSS1, CPA1, SPINK1, CTRC and CFTR genes. ${ }^{34}$ In all patients enrolled in the GOULASH PLUS study, we will analyse the known susceptibility genes associated with $\mathrm{CP}$ in both the trypsin-dependent and misfolding pathological pathways: PRSS1, SPINK1, CTRC and CPA1. In addition, we will also investigate $C F T R$, as certain mutations of this gene may modify pancreatitis risk. ${ }^{35}$ A control cohort of healthy blood donors from the Hungarian National Pancreas Registry will also be analysed to determine the carrier frequency of different variants in this population.

The University of Pécs Medical School will be the leading centre of the study and several laboratory parameters and genetic samples will be measured and processed here. The facilities of the biobank are also located at the University of Pécs Institute for Translational Medicine. The following partners already participate in the GOULASH study: University of Debrecen (Dr Mária Papp), University of Szeged (Dr László Czakó) and hospitals of Gyula (Dr János Novák) and Székesfehérvár (Dr Ferenc Izbéki). The GOULASH PLUS study is open for centres who take part in the GOULASH study.

\section{Duration}

The pilot part of the GOULASH PLUS study started at 1 February 2018 (one year after the first patient was involved in GOULASH study and discharged from the hospital). The estimated date of finishing the study is 28 February 2025.

\section{Cost-effectiveness analysis}

Cost-effectiveness analysis is about the evaluation of the joint distribution of differences in cost and clinical effect. It provides an early opportunity to produce reliable estimates of cost-effectiveness at low marginal cost. Access to individual patient data also permits a wide range of statistical and econometric techniques, to examine the relationship between events of interest and health-related quality of life or to explore subgroup differences. For this purpose along with the clinical outcome measures, we should also gather information about the quality of life of patients and the resource use and unit cost of intervention and treatments used (table 2).

The categories of resource use that are included in the study were determined according to the perspective of the payer. This perspective usually includes direct medical care, including the intervention itself, treatment of any side effects or complications, and follow-up care to the intervention or the underlying condition, which in this case is complemented with a payment for sick leave, which is an indirect cost.

Additional information will be required from the patient questionnaire and from the database of the National Health Insurance Fund Administration (NHIFA) and Central Administration of National Pension Insurance.
Table 2 Evaluation of planned categories for costeffectiveness analysis. ER, emergency room; GP, general practitioner; NHIFA, National Health Insurance Fund Administration.

\begin{tabular}{ll}
\hline Domain name & Source of data \\
\hline Health outcomes & \\
\multicolumn{1}{c}{ Mortality } & Civil registration database \\
\hline $\begin{array}{l}\text { Resource utilisation during follow-up period (after discharge) } \\
\text { GP utilisation }\end{array}$ & NHIFA database \\
Outpatient care utilisation & NHIFA database \\
ER utilisation & NHIFA database \\
Hospital readmission & NHIFA database \\
\hline Drug utilisation & NHIFA database \\
\hline Indirect cost & \\
\hline Sick leave & Central Administration of \\
& National Pension Insurance \\
\hline
\end{tabular}

\section{Examined parameters}

The following parameters will be measured or collected at $1,2,3,4,5$ and 6 years control:

1. Laboratory parameters:

a. General: amylase $(\mathrm{U} / \mathrm{L})$; lipase $(\mathrm{U} / \mathrm{L})$; white cell count $(\mathrm{G} / \mathrm{L})$; red cell count $(\mathrm{T} / \mathrm{L})$; haemoglobin $(\mathrm{g} / \mathrm{L})$; haematocrit $(\%)$; thrombocyte $(\mathrm{G} / \mathrm{L})$; C-reactive protein $(\mathrm{mg} / \mathrm{L})$; creatinine $(\mu \mathrm{mol} / \mathrm{L})$, carbamid $(\mathrm{mmol} / \mathrm{L})$; serum bilirubin $(\mu \mathrm{mol} / \mathrm{L})$; GGT $(\mathrm{mmol} / \mathrm{L})$; GOT $(\mathrm{mmol} / \mathrm{L})$; GPT $(\mathrm{mmol} / \mathrm{L})$; ALP $(\mathrm{mmol} / \mathrm{L})$; uric acid $(\mathrm{mmol} / \mathrm{L})$; cholesterol (HDL, LDL) $(\mathrm{mmol} / \mathrm{L})$; triglycerides $(\mathrm{mmol} / \mathrm{L})$; serum protein $(\mathrm{g} / \mathrm{L})$; albumin $(\mathrm{g} / \mathrm{L})$.

b. Endocrine: C-peptide* (pmol/L); HgbA1C (\%); OGTT test glucose $(\mathrm{mmol} / \mathrm{L})$ and insulin level (pmol/L) at 0, 60 and $120 \mathrm{~min}$.

c. Exocrine: Faecal elastase test*.

2. Biobank: (a) plasma; (b) serum; (c) stool for later analysis

a. Leptin* (pmol/L); IL-1 $\beta^{*} \quad(\mathrm{pmol} / \mathrm{L}) ;$ IL-6* $(\mathrm{pmol} / \mathrm{L}) ; \quad \mathrm{TNF}-\alpha^{*} \quad(\mathrm{pmol} / \mathrm{L}) ;$ somatostatin* (pmol/L); pancreatic polypeptide* $(\mathrm{pmol} / \mathrm{L})$; amylin* (pmol/L); glucagon* (pmol/L); *signed parameters will be measured at our centre if there is no possibility in the smaller centres.

3. Information about (b) smoking; (c) alcohol; (d) drugs; (e) complains: pain and quality of life-MOS 36-Item Short-Form Health Survey (SF-36); (f) dietary fat intake-DHQ questionnaire; (g) body weight, body mass index (BMI).

4. Genetic: PRSS1, CPA1, SPINK1, CTRC and CFTR.

\section{Primary eendpoint}

The primary endpoint was the development of chronic pancreatitis based on morphological changes and clinical signs. 


\section{Secondary endpoints}

Secondary endpoints include (1) recurrent AP based on the International Association of Pancreatology/American Pancreatic Association (IAP/APA) guideline's 2 out of 3 rule: (a) upper abdominal pain; (b) serum amylase or lipase level more than three times than the upper limit of a normal range; (c) distinctive pancreatic findings on US or CT imaging; (2) exocrine pancreatic insufficiencyfaecal elastase test; (3) endocrine pancreatic insufficiency (based on laboratory test, OGTT and FPG); (4) need for radiological/surgical interventions-medical records; (5) dietary intake assessment-DHQ; (6) quality of life, stress, physical activity questionnaires; (7) pain questionnaires; (8) development of pancreas tumour-medical records; (9) laboratory parameters-inflammatory cytokines, pancreatic peptides and so on (table 1).

\section{The trial organisation, committees and boards}

The coordinator and designer of the GOULASH PLUS study are the Centre for Translational Medicine at the University of Pécs Medical School (coordinating institution and sponsor) and the Hungarian Pancreatic Study Group (HPSG-coordinating society). The HPSG was established in 2011 to stimulate research in pancreatic diseases.

The following committees and boards will be involved:

Steering Committee (SC): The committee will be led by PH (corresponding and principal investigator, a gastroenterologist and internal medicine specialist).

The members in Szeged will be LC (gastroenterologist); Debrecen: MP (gastroenterologist); Pécs: ÁV (gastroenterologist), ON (endocrinologist), BB (endocrinologist), NF (radiologist), OF (radiologist), RP (surgeon), DK (surgeon); Székesfehérvár: FI (gastroenterologist); Gyula: JN (gastroenterologist) and one member of each joined institution. AM is a principal organiser, KM is a trial management specialist, whereas AS leads the multidisciplinary core facility which will assist the scientists to run the study successfully.

The SC will make decisions concerning all relevant questions including dropouts during the study.

International Translational Advisory Board (ITAB): The board will consist of a gastroenterologist (MML), a surgeon and two basic scientists (JPN, MST, OHP).

The ITAB will continuously monitor the progress of the study and will advise the SC.

The study was designed by the SC and ITAB. It was funded by the University of Pécs, Medical School. The sponsor was not involved in the design of the study and will have no access to the database or the randomisation code. The study also contains an independent physician and safety manager.

\section{Study population}

All patients enrolled in the GOULASH study will be offered the opportunity to participate in the GOULASH PLUS study. They will be given detailed verbal and written information by clinical doctors and administrators. After the consent is given, the annual follow-ups will be arranged and the above-detailed tests (table 1) and investigations will be performed (figures 1 and 2).

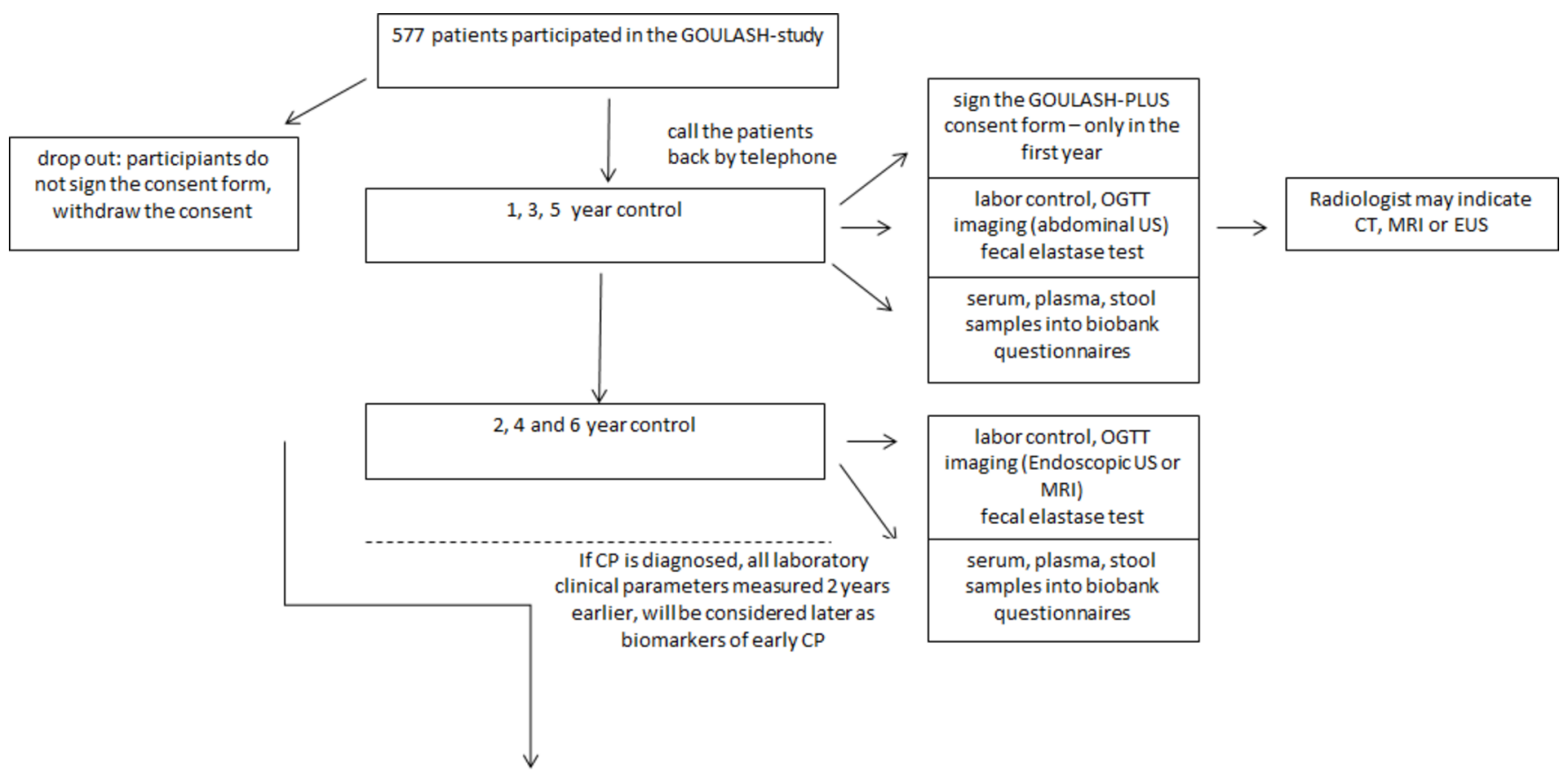

$\mathrm{CP}$ is diagnosed

Figure 1 Flow chart of participants in the GOULASH PLUS study. CP, chronic pancreatitis; EUS, endoscopic ultrasonography; OGTT, oral glucose tolerance test; US, ultrasonography. 


\begin{tabular}{|c|c|c|c|c|c|c|c|}
\hline & GOULASH & \multicolumn{6}{|c|}{ GOULASH-PLUS STUDY PERIOD } \\
\hline & Enrolment* & \multicolumn{6}{|c|}{ Allocation** and follow up visits } \\
\hline TIMEPOINT & $-t_{3} \rightarrow-t_{1}$ & 1 year* & 2 years* & 3 years $*$ & 4 years* & 5 years $*$ & 6 years* \\
\hline \multicolumn{8}{|l|}{ ENROLMENT: } \\
\hline Eligibility screen & $x$ & & & & & & \\
\hline $\begin{array}{r}\text { Informed GOUALSH } \\
\text { consent }{ }^{* * *} \\
\end{array}$ & $x$ & & & & & & \\
\hline Discharge from hospital & $x$ & & & & & & \\
\hline Allocation & & $x$ & & & & & \\
\hline \multicolumn{8}{|l|}{ ASSESSMENTS: } \\
\hline GOULASH-PLUS consent & & $x$ & & & & & \\
\hline Labor control & & $x$ & $x$ & $x$ & $x$ & $x$ & $x$ \\
\hline OGTT**** & & $x$ & $x$ & $x$ & $x$ & $x$ & $x$ \\
\hline $\begin{array}{r}\text { Imaging } \\
\text { (abdominal US) }{ }^{* * * * * *} \\
\end{array}$ & & $x$ & & $x$ & & $x$ & \\
\hline $\begin{array}{r}\text { Imaging } \\
\text { (endoscopic US) } \\
\end{array}$ & & & $x$ & & $x$ & & $x$ \\
\hline Fecal elastase test & & $x$ & $x$ & $x$ & $x$ & $x$ & $\mathrm{x}$ \\
\hline $\begin{array}{r}\text { Biobank (serum, } \\
\text { plasma, stool) } * * * * * *\end{array}$ & & $x$ & $x$ & $x$ & $x$ & $x$ & $x$ \\
\hline Questionnaires $* * * * * * *$ & & $x$ & $x$ & $x$ & $x$ & $x$ & $x$ \\
\hline Genetic $* * * * * * * *$ & & $x$ & & & & & \\
\hline
\end{tabular}

Figure 2 Shows the schedule of enrolment and assessments of participants according to the SPIRIT 2013 guideline. *A total of 957 patients participated in the GOULASH study. ${ }^{* \star T h e ~ A d m i n i s t r a t o r ~ w i l l ~ c a l l ~ t h e ~ p a t i e n t ' s ~ a t t e n t i o n ~ t o ~ f o l l o w-u p ~ v i s i t s ~ b y ~}$ telephone. ${ }^{* *}$ Based on the preliminary data concerning the compliance with the 1-month follow-up, we estimate dropouts and participants who do not sign the consent form. ${ }^{* \star \star *}$ Oral glucose tolerance test (OGTT) will be performed if diabetes mellitus is unknown and the fasting plasma glucose is level is $\leq 7 \mathrm{mmol} / \mathrm{L}$. ${ }^{* \star \star \star \star} B$ Based on morphological changes, the radiologist may recommend CT, endoscopic ultrasonography or MRI examination. ${ }^{* \star \star \star \star \star I n ~ t h e ~ U n i v e r s i t y ~ o f ~ P e ́ c s, ~ s a m p l e s ~ i n ~ t h e ~ b i o b a n k ~ w i l l ~}$ be kept to measure any kind of possible biomarkers which could be a candidate in the diagnosis of early phase of chronic pancreatitis. ${ }^{\star \star \star \star \star \star \star \star} T$ The questionnaires will be completed by the clinical research administrators. ${ }^{* \star \star \star \star \star \star \star}$ It will be performed in the University of Pécs and Szeged.

\section{Inclusion criteria for GOULASH study}

The inclusion criteria are as follows: (1) patients above 18 years, (2) diagnosed AP based on the ' 2 out of 3 ' rules of IAP/APA guideline ${ }^{36}$ : (a) upper abdominal pain,(b) serum amylase or lipase $>3 \times$ upper limit of normal range and (c) characteristic findings on pancreatic imaging; however, those patients without abdominal pain will be excluded because the onset of AP cannot be assessed; (3) written informed consent form is signed.

\section{Exclusion criteria or reasons of compulsory dropout for the GOULASH study}

The exclusion criteria are as follows: (1) hospitalisation 72 hours before admission; (2) abdominal pain >120 hours (5 days); (3) delirium tremens; (4) Child-Pugh C stage liver cirrhosis; (5) AP due to malignancy; (6) already on artificial nutrition (Enteral nutrition (EN) or parenteral nutrition (PN)); (7) pregnancy; (8) BMI above 40 or below 18; (9) age above 80; (10) ketoacidosis; (11) whenever CT with contrast is contraindicated.

\section{Inclusion criteria for GOULASH PLUS}

Patients participated in the GOULASH study and signed the consent form.

\section{Exclusion criteria for GOULASH PLUS}

The patient does not sign the consent form, or the patient withdraws the consent.

According to the ethical laws, individuals can leave the study at any time without reason and explanation. Serious medical reasons not related to pancreatitis (ie, accidents, stroke) means withdrawal of the subject from the study. All withdrawals will be filled; moreover, there will be no replacement of individual subjects after withdrawal.

\section{Sample size}

Sample size calculation for the GOULASH study was based on data extracted from the Hungarian National Registry of AP operated by the HPSG. The calculated sample size of the GOULASH study is 957 participants; however, the sample size calculation for the GOULASH PLUS study was performed based on the data of the HPSG registry and the articles published by Yadav et $a \tilde{e}$ and Kin $e t a l^{37}$ Seventy-five newly developed CP is necessary to reach the required $5 \%$ alpha and $80 \%$ power for the individual biomarkers. Literature data suggest that the chance to develop CP is around $13 \%$ within the 6-year period. Therefore, the GOULAS-PLUS study requires 577 patients involvement. Several biomarkers including laboratory and imaging parameters, 2 years prior to the development of $\mathrm{CP}$, will be investigated. Based on the preliminary data concerning 
the compliance with the 1 month follow-up, we estimate that $80 \%$ of the GOULASH study cohort will take part in the GOULASH PLUS study. However, we expect that the number of patients will decrease every year due to multiple reasons such as loss of motivation, migration and other reasons independent of AP (ageing, comorbidity).

Pitfalls: If the number of participants from the GOULASH study is low, patients with AP who were not included the GOULASH study will be approached too. However, in that case, the same inclusion criteria are kept, which are written in the GOULASH protocol.

\section{Data management and statistical probes \\ Data handling}

Data will be handled by Data Management and Biostatistics provider Organisation of the Centre for Translational Medicine. Electronic case report form (eCRF) will be used. The investigator will ensure that the data in the eCRF are accurate, complete and legible. Detailed data flow will be described in the data management plan. Any missing, impossible or inconsistent recordings in the eCRFs will be referred back to the investigator using a data query form, and be documented for each individual subject before clean file status is declared.

\section{Study populations}

Two analysis populations will be defined: (1) the safety analysis set consists of all patients enrolled to the study; (2) per protocol set with all enrolled patients who finished the study conforming to the requirements of the study protocol.

\section{Applied software}

Statistical analysis will be performed using SAS V.9.2 or SPSS V.24 statistical packages; Microsoft MS Word 2010 will be used for reporting.

\section{Statistical methods}

Baseline patient and disease characteristics will be analysed using descriptive analysis. Demographic and baseline characteristics will be summarised for the overall study population. Continuous variables will be described by mean, median, SD and ranges, and categorical variables will be described by absolute and relative frequencies. A graphical presentation of efficacy variables will be prepared, if applicable.

Descriptive statistics for the followed parameters will be analysed. Mean changes (and their 95\% CI) from baseline to end-of-study visit will be presented as well. $\chi^{2}$ tests will be applied to compare proportions between the different groups. Mortality will be investigated using the KaplanMeier survival analysis method, while subgroup comparisons will be performed using the $\chi^{2}$ or Fisher's exact test, as appropriate.

\section{Subgroups}

The following subgroups will be made during statistical analyses: (1) ages (under 40, 40-60, 60-80, above 80 years); (2) BMI (below 20, 20-25, 25-30, 30-35, above 35); (3) the number of RAP, CP; (4) severity of the disease SAP (severe AP) and MAP (mild and moderately severe AP).

All subgroup analyses will be done descriptively. No confirmatory statistical testing will be applied. Hence, statistical tests and the $p$ values attached to them will be regarded as descriptive and not as tests of hypotheses.

Details of the applied statistical tests will be described in the statistical analysis plan.

\section{Patient and public involvement statement}

The GOULASH PLUS study aims to detect the first signs and biomarkers of early CP. It will be a 6-year observational prospective follow-up study with no intervention. The study will provide results, which will help to understand the development and risk factors of late complications in AP and the development of the pancreatic damage. All patients enrolled in the GOULASH study will be offered the opportunity to participate in the GOULASH PLUS study. They will be given detailed verbal and written information by clinical doctors and administrators. We would like to publish the results in one of the internationally highly recognised journals.

\section{PUBLICATION POLICY}

Centres providing more than 25 patients can provide two authors to the author's list. Every additional 25 patients will give the opportunity to nominate an author.

\section{DISCUSSION}

\section{Expected results}

We aim to understand the development and natural history of CP at its early phase. We would like to identify factors that are associated with the development of CP. With the help of the new information, new pharmaceutical or lifestyle interventions could be recognised. Our aim was to publish our future data in a high ranking multidisciplinary or gastroenterology journal, which of course will largely depend on the outcome of the study. We have done all the necessary administrative duties, which are compulsory for such distinguished clinical journals.

\section{Author affiliations}

${ }^{1}$ Institute for Translational Medicine, University of Pécs, Medical School, Pécs, Hungary

${ }^{2}$ Division of Gastroenterology, First Department of Medicine, University of Pécs, Pécs, Hungary

${ }^{3}$ Gastroenterological Clinic, Faculty of Medicine, Slovak Medical University in Bratislava, Bratislava, Slovakia

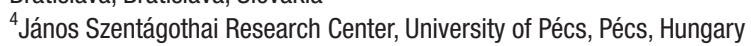

${ }^{5}$ Division of Endocrinology and Metabolism, First Department of Medicine, University of Pécs, Pécs, Hungary

${ }^{6}$ Department of Radiology, Medical School, University of Pécs, Pécs, Hungary

${ }^{7}$ Surgery Clinic, University of Pécs, Pécs, Hungary

${ }^{8}$ First Department of Medicine, University of Szeged, Szeged, Hungary

${ }^{9}$ Department of Internal Medicine, Division of Gastroenterology, University of Debrecen, Debrecen, Hungary

${ }^{10}$ First Department of Gastroenterology, Szent György University Teaching Hospital of Fejér County, Székesfehérvár, Hungary 
${ }^{11}$ First Department of Gastroenterology, Pándy Kálmán Hospital of Békés County, Gyula, Hungary

${ }^{12}$ Department of Molecular and Cell Biology, Henry M Goldman School of Dental Medicine Boston University, Boston, Massachusetts, UK

${ }^{13}$ Department of Medicine A, Universitatsmedizin Greifswald, Greifswald, Germany

${ }^{14}$ Cancer Research UK Liverpool Cancer Trials Unit, University of Liverpool, Liverpool, UK

${ }^{15}$ Schoool of Biosciences, Cardiff University, Cardiff, UK

${ }^{16}$ Hungarian Academy of Sciences- University of Szeged, Momentum

Gastroenterology Multidisciplinary Research Group, Szeged, Hungary

Acknowledgements We gratefully thank Vivien Vass and Ivett Szomor, clinical researcher administrators, for their work and filling out the questionnaires with the patients.

Collaborators Centres and team members: The University of Pécs Medical School will be the leading centre of the study and several laboratory parameters and genetic samples will be measured and processed here. The facilities of the biobank are also located at the University of Pécs Institute for Translational Medicine. The coordinator anddesigner of the GOULASH Plus study is the HPSG (Hungarian Pancreatic Study Group). The following partners already participate in the GOULASH study; University of Debrecen (Dr Mária Papp), University of Szeged (Dr László Czakó) and hospitals of Gyula (Dr János Novák) and Székesfehérvár (Dr Ferenc Izbéki). The GOULASH-PLUS study is open for centres who take part in the GOULASH study.

Contributors All authors were involved in the study design, edited the manuscript, read and approved the final manuscript. During the study AM, BE, DP, PHJr, PS and PH will manage the treatment of the patients. BE, PS and ÁV will do the endoscopic ultrasonography examination. NF and OF will perform the abdominal ultrasonography of the patients. DK and RP will operate the patients if it is necessary. EH will perform the genetical testing of patients. MP, FI, LC, JN and LG will be responsible for managing the study in other centers. ML, JN, MST and OHP are members of ITAB. MK, BB, ON and AS will be members of the SC. PH, KM and AM drafted the manuscript.

Funding Centre costs (IT, biostatistics, trial organisation, and so on) are covered by the University of Pécs Medical School, Momentum Grant of the Hungarian Academy of Sciences (LP2014-10/2014), Highly Cited Publication Grant (KH 125678) of the National Research Development and Innovation Office (GINOP 2.3.2-15-201600048 Stay Alive) and (EFOP 3.6.2-16-2017-00006 Live Longer), Grant of the University of Pécs (KA-2019-14 to AM) and Translational Medicine Foundation. Since no additional treatment is necessary for the study, the general healthcare costs are covered by the National Healthcare System. This study was designed with help of the Centre for Translational Medicine at the University of Pécs. This centre is committed to improving patients' life with research activities such as registries, observational and interventional trial organisations (https://tm-centre.org).

Competing interests None declared.

Patient consent for publication Obtained.

Ethics approval The study will be performed in accordance with the Declaration of Helsinki and the principles of ICH-GCP guidelines. The ethical approval from ETT-TUKEB, Secretary of Medical Research Council Scientific and Research Ethics Committee is accepted (5753-2/2018/EKU).

Provenance and peer review Not commissioned; externally peer reviewed.

Open access This is an open access article distributed in accordance with the Creative Commons Attribution Non Commercial (CC BY-NC 4.0) license, which permits others to distribute, remix, adapt, build upon this work non-commercially, and license their derivative works on different terms, provided the original work is properly cited, appropriate credit is given, any changes made indicated, and the use is non-commercial. See: http://creativecommons.org/licenses/by-nc/4.0/.

\section{REFERENCES}

1. Sand J, Nordback I. Acute pancreatitis: risk of recurrence and late consequences of the disease. Nat Rev Gastroenterol Hepatol 2009;6:470-7.

2. Párniczky A, Kui B, Szentesi A, et al. Prospective, multicentre, nationwide clinical data from 600 cases of acute pancreatitis. PLoS One 2016;11:e0165309.

3. Nikkola J, Rinta-Kiikka I, Räty S, et al. Pancreatic morphological changes in long-term follow-up after initial episode of acute alcoholic pancreatitis. J Gastrointest Surg 2014;18:164-71. discussion 170-1.
4. Ahmed Ali U, Issa Y, Hagenaars JC, et al. Risk of recurrent pancreatitis and progression to chronic pancreatitis after a first episode of acute pancreatitis. Clin Gastroenterol Hepatol 2016;14:738-46.

5. Yadav D, O'Connell M, Papachristou GI. Natural history following the first attack of acute pancreatitis. Am J Gastroenterol 2012;107:1096-103.

6. Puylaert M, Kapural L, Van Zundert J, et al. 26. Pain in chronic pancreatitis. Pain Pract 2011;11:492-505.

7. Kleeff J, Whitcomb DC, Shimosegawa T, et al. Chronic pancreatitis. Nat Rev Dis Primers 2017;3:17060.

8. Takács T, Czakó L, Dubravcsik Z, et al. [Chronic pancreatitis. Evidence based management guidelines of the Hungarian Pancreatic Study Group]. Orv Hetil 2015;156:262-88.

9. Pezzilli R. Etiology of chronic pancreatitis: has it changed in the last decade? World J Gastroenterol 2009;15:4737-40.

10. Durbec JP, Sarles H. Multicenter survey of the etiology of pancreatic diseases. Relationship between the relative risk of developing chronic pancreaitis and alcohol, protein and lipid consumption. Digestion 1978;18:337-50.

11. Löhr JM, Dominguez-Munoz E, Rosendahl J, et al. United European Gastroenterology evidence-based guidelines for the diagnosis and therapy of chronic pancreatitis (HaPanEU). United European Gastroenterol J 2017;5:153-99.

12. Layer P, Keller J. Lipase supplementation therapy: standards, alternatives, and perspectives. Pancreas 2003;26:1-7.

13. Whitcomb DC, Frulloni L, Garg P, et al. Chronic pancreatitis: an international draft consensus proposal for a new mechanistic definition. Pancreatology 2016;16:218-24.

14. Yamabe A, Irisawa A, Shibukawa G, et al. Early diagnosis of chronic pancreatitis: understanding the factors associated with the development of chronic pancreatitis. Fukushima J Med Sci 2017;63:1-7.

15. Marra-Lopez Valenciano C, Bolado Concejo F, Marín Serrano E, et al. Prevalence of exocrine pancreatic insufficiency in patients with chronic pancreatitis without follow-up. PANCR-EVOL Study. Gastroenterol Hepatol 2018;41:77-86.

16. de la Iglesia-Garcia D. Increased risk of mortality associated with pancreatic exocrine insufficiency in patients with chronic pancreatitis. J Clin Gastroenterol 2017.

17. de la Iglesia-García D, Huang W, Szatmary P, et al. Efficacy of pancreatic enzyme replacement therapy in chronic pancreatitis: systematic review and meta-analysis. Gut 2017;66:1354.1-5.

18. Whitcomb DC, Lehman GA, Vasileva G, et al. Pancrelipase delayed-release capsules (CREON) for exocrine pancreatic insufficiency due to chronic pancreatitis or pancreatic surgery: A double-blind randomized trial. Am J Gastroenterol 2010;105:2276-86.

19. Boreham B, Ammori BJ. A prospective evaluation of pancreatic exocrine function in patients with acute pancreatitis: correlation with extent of necrosis and pancreatic endocrine insufficiency. Pancreatology 2003;3:303-8.

20. Rickels MR, Bellin M, Toledo FG, et al. Detection, evaluation and treatment of diabetes mellitus in chronic pancreatitis: recommendations from PancreasFest 2012. Pancreatology 2013;13:336-42.

21. Jivanji CJ, Soo DH, Petrov MS. Towards reducing the risk of new onset diabetes after pancreatitis. Minerva Gastroenterol Dietol 2017;63:270-84

22. Yang CJ, Bliss LA, Schapira EF, et al. Systematic review of early surgery for chronic pancreatitis: impact on pain, pancreatic function, and re-intervention. J Gastrointest Surg 2014;18:1863-9.

23. Dranka-Bojarowska D, Lekstan A, Olakowski M, et al. The assessment of serum concentration of adiponectin, leptin and serum carbohydrate antigen-19.9 in patients with pancreatic cancer and chronic pancreatitis. J Physiol Pharmacol 2015;66:653-63.

24. Komar HM, Hart PA, Cruz-Monserrate Z, et al. Local and systemic expression of immunomodulatory factors in chronic pancreatitis. Pancreas 2017;46:986-93.

25. Cernea S, Dobreanu M. Diabetes and beta cell function: from mechanisms to evaluation and clinical implications. Biochem Med 2013;23:266-80.

26. Hegyi E, Sahin-Tóth M. Genetic risk in chronic pancreatitis: the trypsin-dependent pathway. Dig Dis Sci 2017;62:1692-701.

27. Márta K, Szabó AN, Pécsi D, et al. High versus low energy administration in the early phase of acute pancreatitis (GOULASH trial): protocol of a multicentre randomised double-blind clinical trial. BMJ Open 2017;7:e015874.

28. Møller Andersen A, Malmstrøm ML, Novovic S, et al. [Transabdominal ultrasound examination, contrast-enhanced ultrasound examination and endoscopic ultrasound scanning in the determination of the 
aetiology and the degree of severity in acute pancreatitis]. Ugeskr Laeger 2013;175:1482-4.

29. Bolondi L, Li Bassi S, Gaiani S, et al. Sonography of chronic pancreatitis. Radiol Clin North Am 1989;27:815-33.

30. Ikeda M, Sato T, Morozumi A, et al. Morphologic changes in the pancreas detected by screening ultrasonography in a mass survey, with special reference to main duct dilatation, cyst formation, and calcification. Pancreas 1994;9:508-12.

31. Issa Y, Kempeneers MA, van Santvoort HC, et al. Diagnostic performance of imaging modalities in chronic pancreatitis: a systematic review and meta-analysis. Eur Radiol 2017;27:3820-44.

32. Hoffmeister A, Mayerle J, Beglinger $\mathrm{C}$, et al. English language version of the S3-consensus guidelines on chronic pancreatitis: Definition, aetiology, diagnostic examinations, medical, endoscopic and surgical management of chronic pancreatitis. $Z$ Gastroenterol 2015;53:1447-95.
33. Rana SS, Vilmann P. Endoscopic ultrasound features of chronic pancreatitis: a pictorial review. Endosc Ultrasound 2015;4:10-14.

34. Ravi Kanth V, Nageshwar Reddy D. Genetics of acute and chronic pancreatitis: an update. World J Gastrointest Pathophysiol 2014;5:427-37.

35. Hegyi P, Wilschanski M, Muallem S, et al. CFTR: a new horizon in the pathomechanism and treatment of pancreatitis. Rev Physiol Biochem Pharmacol 2016;170:37-66.

36. Working Group IAP/APA Acute Pancreatitis Guidelines. IAP/APA evidence-based guidelines for the management of acute pancreatitis Pancreatology 2013;13(4 Suppl 2):e1-e15.

37. Lin YC, Kor CT, Su WW, et al. Risk factors and prediction score for chronic pancreatitis: a nationwide population-based cohort study. World J Gastroenterol 2018;24:5034-45. 